\title{
LOWER BOUNDS FOR DIMENSIONS OF SUMS OF SETS
}

\author{
DANIEL M. OBERLIN
}

\begin{abstract}
We study lower bounds for the Minkowski and Hausdorff dimensions of the algebraic sum $E+K$ of two sets $E, K \subset \mathbb{R}^{d}$.
\end{abstract}

\section{INTRODUCTION}

Suppose $E, K \subset \mathbb{R}^{d}$ are compact sets. We are interested in finding lower bounds for the Hausdorff and Minkowski dimensions of the sum set $E+K$ which are better than the trivial lower bound

$$
\operatorname{dim}(E+K) \geq \max (\operatorname{dim}(E), \operatorname{dim}(K)) .
$$

Our general approach will be to fix a "nice" $K$ and to look for some function $\Phi(K, \operatorname{dim}(E))$ such that

$$
\operatorname{dim}(E+K) \geq \Phi(K, \operatorname{dim}(E))>\max (\operatorname{dim}(E), \operatorname{dim}(K))
$$

for all Borel $E \subset \mathbb{R}^{d}$ with $\operatorname{dim}(E)<d$. (There are well-known results for "generic" sums, e.g. the fact, a consequence of [7], that if $E, K \subset \mathbb{R}$, then

$$
\operatorname{dim}(E+t K) \geq \min \{\operatorname{dim}(E)+\operatorname{dim}(K), 1\}
$$

for almost all $t \in \mathbb{R}$. And there are also some interesting results requiring special hypotheses on both $E$ and $K$. One is in [12]: suppose $a \in(0,1 / 2)$ and let $C_{a}$ be the Cantor set

$$
\left\{(1-a) \sum_{j=0}^{\infty} \omega_{j} a^{j}: \omega_{j} \in\{0,1\}\right\} .
$$

Then $\operatorname{dim}\left(C_{a}+C_{b}\right)=\min \left\{\operatorname{dim}\left(C_{a}\right)+\operatorname{dim}\left(C_{b}\right), 1\right\}$ if $\log (b) / \log (a)$ is irrational. See also [14.)

Our observations will fall into two classes, depending on whether dim means Minkowski dimension or Hausdorff dimension.

The case of (upper) Minkowski dimension $\operatorname{dim}_{m}$ is easier and the example of a curve $K$ in $\mathbb{R}^{2}$ appears to be typical here: if $K$ is a line segment then one cannot improve the trivial bound; if $K$ is not a line segment then we will show that

$$
\operatorname{dim}_{m}(E+K) \geq 1+\frac{\operatorname{dim}_{m}(E)}{2} .
$$

Date: August, 2008.

This work was supported in part by NSF grant DMS-0552041. 
It is easy to see that, without further hypotheses on the curve $K$, (1.3) cannot be improved: let $E$ be the cartesian product of two copies of some Cantor set and let $K \doteq K_{0}$ be a curve consisting of a horizontal line segment followed by a vertical one.

Our result for Minkowski dimension will be a generalization of (1.3): we will observe that the lower bound

$$
\operatorname{dim}_{m}(E+K) \geq \operatorname{dim}_{m}(K)+\operatorname{dim}_{m}(E)-\frac{\operatorname{dim}_{m}(K) \operatorname{dim}_{m}(E)}{d}
$$

holds for certain Cantor sets $K \subset \mathbb{R}$ and also whenever $K$ is a $k$-dimensional manifold in $\mathbb{R}^{d}$ which satisfies a certain nondegeneracy condition.

For Hausdorff dimension $\operatorname{dim}_{h}$ we know very little. We will make the trivial observation that if $K \subset \mathbb{R}^{d}$ is a Salem set, then there is the optimal estimate $\operatorname{dim}_{h}(E+K)=\min \left\{\operatorname{dim}_{h}(E)+\operatorname{dim}_{h}(K), d\right\}$. We will show that if $K$ is the "middle thirds" Cantor set in $\mathbb{R}$, then

$$
\operatorname{dim}_{h}(E+K) \geq \frac{1+\operatorname{dim}_{h}(E)}{2}
$$

(which improves the trivial bound only if $\operatorname{dim}_{h}(E)>2 \log 2 / \log 3-1$ ). We will prove a result concerning convolution estimates which yields the analog of (1.4) for a few model surfaces. And we will conclude with some remarks about nondegenerate curves. $\S 3$

We will consider Minkowski dimension in 92 and Hausdorff dimension in

\section{MinKOWSKI DIMENSION}

If $E \subset \mathbb{R}^{d}$ then $\operatorname{dim}_{m}(E) \geq \beta$ is equivalent to

$$
m_{d}(E+B(0, \delta)) \geq C(\epsilon) \delta^{d-\beta+\epsilon}
$$

for $\epsilon>0$ and small $\delta>0$. Our strategy for proving (1.4) depends on the observation that (1.4) is implied by the estimate, to hold for all for Borel $F \subset \mathbb{R}^{d}$

$$
m_{d}(F+K) \geq C(K) m_{d}(F)^{1-\alpha / d}
$$

where $\alpha=\operatorname{dim}_{m}(K)$ : if (2.2) holds and $\operatorname{dim}_{m}(E) \geq \beta$, then (2.1) implies that

$$
m_{d}(E+K+B(0, \delta)) \gtrsim \delta^{d-(\alpha+\beta-\alpha \beta / d)+\epsilon^{\prime}}
$$

where $\epsilon^{\prime}=\epsilon(1-\alpha / d)$. Inequalities (2.2) were the subject of the two papers [10] and [11, and we will rely on results and ideas from those papers as we verify (1.4) for various Cantor sets and $k$-surfaces.

The results of [11] imply inequalities (2.2) for certain Cantor sets $K$. To describe them we establish some notation. Fix a positive integer $n \geq 3$ and let $G(n)=\{0,1, \ldots, n-1\}$, which we will interpret as either a set of integers or as a realization of the group of integers modulo $n$. Fix a subset $S \subset G(n)$ such that $0 \in S$ and consider the generalized Cantor set $K \subset[0,1]$ consisting of all sums $\sum_{j=1}^{\infty} s_{j} n^{-j}$ such that each $s_{j} \in S$. Then the Hausdorff 
and Minkowski dimensions of $K$ are both equal to $\log (|S|) / \log (n)$. Let $\widetilde{m}_{n}$ be normalized counting measure on $G(n)$ and $\widetilde{m}$ be Lebesgue measure on $[0,1)$ (which, when equipped with addition modulo 1 , we regard here as a realization of the circle group). Theorem 2 of [11] states that if $\gamma \in(0,1)$ and the inequality

$$
\widetilde{m}_{n}(E+S) \geq \widetilde{m}_{n}(E)^{\gamma}
$$

holds for $E \subset G(n)$, where the addition is in the group $G(n)$, then the inequality

$$
\widetilde{m}(E+K) \geq \widetilde{m}(E)^{\gamma}
$$

holds for $E \subset[0,1)$, with addition modulo 1 . Unwrapping the addition modulo 1 , this implies

$$
m_{1}(F+K) \geq m_{1}(F)^{\gamma} / 2
$$

for Borel $F \subset \mathbb{R}$. It is easy to verify that (2.3) holds if $|S|=n-1$ and $\gamma=1-\log (|S|) / \log (n)$ (the case $n=3$ yielding the classical Cantor set). Thus, for these Cantor sets $K,(2.2)$ and so (1.4) hold. There are also a few other cases where (2.2) and (1.4) are true, $n=5$ and $|S|=2$ giving one example. But the method of [11] is not very flexible and, in particular, does not seem to be up to solving the interesting problem of establishing (2.2) for all of the Cantor sets $C_{a}$ mentioned in the introduction.

We now consider the situation when $K$ is a $k$-surface in $\mathbb{R}^{d}$. The simplest case is when $K$ is a curve in $\mathbb{R}^{2}$. As previously mentioned, if $K$ is a line segment - which in this setting we consider to be a degenerate curve - we cannot improve on the trivial bound (1.1). If $K$ is not a line segment, an estimate of the form (2.2) for $K$ follows from the more general estimate (Theorem B in [10]):

$$
\sqrt{m_{d}(K-K) m_{d}(E)} \leq m_{d}(K+E) .
$$

To be specific, if the curve $K$ is nondegenerate, i.e., not a line segment, then $m_{2}(K-K)>0$ and so (2.4) gives (2.2) with $\alpha=1, d=2$. This, in turn, gives (1.3).

A first step towards generalizing (1.3) is to find an appropriate notion of nondegeneracy for a $k$-surface $K$ in $\mathbb{R}^{d}$. We will refer to a mapping $\Psi: K^{d} \rightarrow\left(\mathbb{R}^{d}\right)^{k}$ of the form

$$
\Psi:\left(x_{1}, \ldots, x_{d}\right) \mapsto\left(\sum_{j=1}^{d} \tau_{j}^{1} x_{j}, \ldots, \sum_{j=1}^{d} \tau_{j}^{k} x_{j}\right), \tau_{j}^{k} \in\{-1,1\}
$$

as an inflation map (the word "inflation" in this context comes from [3]). For example, if $k=1, d=2$, then $\Psi\left(x_{1}, x_{2}\right)=x_{1}-x_{2}$ is an inflation map. We will consider the $k$-surface $K \subset \mathbb{R}^{d}$ to be nondegenerate if there is an inflation map $\Psi$ such that $\Psi\left(K^{d}\right)$ has positive Lebesgue measure in $\left(\mathbb{R}^{d}\right)^{k}$. We would like to prove that if $K$ is a nondegenerate $k$-surface in $\mathbb{R}^{d}$, then (2.2) holds with $\alpha=k$, and so (1.4) holds. Unfortunately, the proofs that we have are tied to particular inflation maps. Thus, in particular, we do not 
even know whether there is an analog of (2.4) with $K+K$ in place of $K-K$. The current situation is most satisfactory when $k=d-1$ : Proposition 4 in [10] shows that if

$$
\left\{\left(x_{1}-x_{2}, \ldots, x_{1}-x_{d}\right): x_{j} \in K\right\}
$$

has positive Lebesgue measure in $\left(\mathbb{R}^{d}\right)^{d-1}$, then (2.2) holds with $\alpha=d-1$. Thus (1.4) holds.

The results of 10 were phrased in terms of a particular inflation map for $k$-surfaces in $\mathbb{R}^{d}$ :

$\Psi_{0}:\left(x_{1}, \ldots, x_{d}\right) \mapsto\left(x_{1}+\cdots+x_{l}-x_{l+1}, \ldots, x_{1}+\cdots+x_{l}-x_{d}\right), l=d-k$.

Unfortunately (and unfortunately unnoticed when [10] was written), unless $k=1$ or $k=d-1$ or $d \leq 4$, if $K$ is a reasonable $k$-surface in $\mathbb{R}^{d}$, then $\Psi_{0}$ can never map $K^{d}$ onto a set of positive measure in $\left(\mathbb{R}^{d}\right)^{k}$ : roughly, the term $x_{1}+\cdots+x_{l}$ uses up $l k$ dimensions but can make a contribution of only $d$ dimensions to the range of $\Psi_{0}$, and $l k=(d-k) k \leq d$ forces $k=1$ or $k=d$ unless $d \leq 4$. To rule out such $\Psi$ we now add another condition to the definition of inflation map: suppose $\left\{e_{1}, \ldots, e_{d}\right\}$ is the usual basis for $\mathbb{R}^{d}$ and define $K_{0} \subset \mathbb{R}^{d}$ by

$$
K_{0}=\bigcup_{\left\{1 \leq i_{1}<\cdots<i_{k} \leq d\right\}}\left\{a_{i_{1}} e_{i_{1}}+\cdots+a_{i_{k}} e_{i_{k}}:-\frac{1}{2} \leq a_{i_{j}} \leq \frac{1}{2}\right\}
$$

so that $K_{0}$ is $k$-dimensional and analogous to the curve $K_{0}$ mentioned after (1.3). Then an inflation map is (re)defined to be a map $\Psi$ of the form (2.5) for which $\Psi\left(\left(K_{0}\right)^{d}\right)$ has positive Lebesgue measure in $\left(\mathbb{R}^{d}\right)^{k}$. Here is an example (and for the remainder of this paper, with $k$ and $d$ fixed, $\Psi$ will stand for this particular example). Write $d=q k+r$ with $q, r$ nonnegative integers and $0 \leq r<k$. Define $\Psi$ by

$$
\Psi: x=\left(x_{1}, \ldots, x_{d}\right) \mapsto\left(\psi_{1}(x), \ldots, \psi_{k}(x)\right)
$$

where, for $1 \leq j \leq k$, we choose $n_{j} \in\{0,1, \ldots, r-1\}$ such that

$$
n_{j} k<j r \leq\left(n_{j}+1\right) k
$$

and then set

$\psi_{j}(x)=\left(x_{d}+x_{d-1}+\cdots+x_{d-n_{j}}\right)+\left(x_{j q}+x_{j q-1}+\cdots+x_{j q-(q-2)}-x_{j q-(q-1)}\right)$.

For example, if $d=5$ and $k=2$, then

$$
\Psi\left(x_{1}, \ldots, x_{5}\right)=\left(x_{5}+x_{2}-x_{1}, x_{5}+x_{4}-x_{3}\right) .
$$

Also, define $\psi_{j}^{\prime}$ and $\psi_{j}^{\prime \prime}$ by

$\psi_{j}^{\prime}(x)=x_{j q}+x_{j q-1}+\cdots+x_{j q-(q-2)}-x_{j q-(q-1)}, \psi_{j}^{\prime \prime}(x)=x_{d}+x_{d-1}+\cdots+x_{d-n_{j}}$

and

$$
\Psi^{\prime}(x)=\left(\psi_{1}^{\prime}(x), \ldots, \psi_{k}^{\prime}(x)\right), \Psi^{\prime \prime}(x)=\left(\psi_{1}^{\prime \prime}(x), \ldots, \psi_{k}^{\prime \prime}(x)\right)
$$


With a view towards Lemma 2.2 below, as well as to establish that $\Psi\left(\left(K_{0}\right)^{d}\right)$ has positive Lebesgue measure in $\left(\mathbb{R}^{d}\right)^{k}$, we now indicate how to construct probability measures $\lambda_{1}, \ldots, \lambda_{d}$ on $K_{0}$ such that

$$
\int_{K_{0}} \cdots \int_{K_{0}} f\left(\Psi\left(x_{1}, \ldots, x_{d}\right)\right) d \lambda_{1}\left(x_{1}\right) \cdots d \lambda_{d}\left(x_{d}\right) \leq \int_{\left(\mathbb{R}^{d}\right)^{k}} f d m_{d k}
$$

for nonnegative functions $f$ on $\left(\mathbb{R}^{d}\right)^{k}$. Each of the $\lambda_{j}$ 's will be $k$-dimensional Lebesgue measure on one of the sets

$$
\left\{a_{i_{1}} e_{i_{1}}+\cdots+a_{i_{k}} e_{i_{k}}:-\frac{1}{2} \leq a_{i_{j}} \leq \frac{1}{2}\right\}
$$

from (2.6).

To give the idea, we first treat the case $d=5$ and $k=2$ mentioned above. Choose $\lambda_{1}$ and $\lambda_{2}$ so that, if $g$ is a function on $\mathbb{R}^{5}$, then

$$
\int_{K_{0}} \int_{K_{0}} g\left(x_{2}-x_{1}\right) d \lambda_{2}\left(x_{2}\right) d \lambda_{1}\left(x_{1}\right)=\int_{-1 / 2}^{1 / 2} \cdots \int_{-1 / 2}^{1 / 2} g\left(0, a_{2}^{1}, a_{3}^{1}, a_{4}^{1}, a_{5}^{1}\right) d a_{2}^{1} \cdots d a_{5}^{1}
$$

and then $\lambda_{3}$ and $\lambda_{4}$ so that

$$
\int_{K_{0}} \int_{K_{0}} g\left(x_{4}-x_{3}\right) d \lambda_{4}\left(x_{4}\right) d \lambda_{3}\left(x_{3}\right)=\int_{-1 / 2}^{1 / 2} \ldots \int_{-1 / 2}^{1 / 2} g\left(a_{1}^{2}, 0, a_{3}^{2}, a_{4}^{2}, a_{5}^{2}\right) d a_{1}^{2} d a_{3}^{2} d a_{4}^{2} d a_{5}^{2} .
$$

Then choose $\lambda_{5}$ so that

$$
\int_{K_{0}} g\left(x_{5}\right) d \lambda_{5}\left(x_{5}\right)=\int_{-1 / 2}^{1 / 2} \int_{-1 / 2}^{1 / 2} g\left(b_{1,1}, b_{1,2}, 0,0,0\right) d b_{1,1} d b_{1,2} .
$$

Clearly

$$
\int_{K_{0}} \cdots \int_{K_{0}} f\left(x_{5}+x_{2}-x_{1}, x_{5}+x_{4}-x_{3}\right) d \lambda_{1}\left(x_{1}\right) \cdots d \lambda_{5}\left(x_{5}\right) \leq \int_{\left(\mathbb{R}^{5}\right)^{2}} f d m_{10}
$$

for nonnegative functions $f$ on $\mathbb{R}^{5} \times \mathbb{R}^{5}$, giving (2.9).

In the general case we write $\left(a_{1}^{1}, \ldots, a_{d}^{1} ; a_{1}^{2}, \ldots, a_{d}^{2} ; \ldots ; a_{1}^{k}, \ldots, a_{d}^{k}\right)$ for an element of $\left(\mathbb{R}^{d}\right)^{k}$ and split the variables $a_{i}^{j}$ into two classes. For a nonnegative integer $i$, let $[i]$ satisfy $1 \leq[i] \leq d$ and $[i]=i \bmod d$. For $1 \leq j \leq k$ we will say that the $r$ variables $a_{[(j-1) r+1]}^{j}, a_{[(j-1) r+2]}^{j}, \ldots, a_{[j r]}^{j}$ are in the second class and the remaining $k q=d-r$ variables $\left\{a_{p_{j, n}^{j}}^{j}\right\}_{n=1}^{k q}$ are in the first class.

Choose the first $k q$ of the measures $\lambda_{i}$ so that, for $1 \leq j \leq k$

$$
\int_{\left(K_{0}\right)^{q}} g\left(\psi_{j}^{\prime}\left(x_{(j-1) q+1}, \ldots, x_{j q}\right)\right) d \lambda_{j q}\left(x_{j q}\right) \cdots d \lambda_{(j-1) q+1}\left(x_{(j-1) q+1}\right)
$$

is equal to

$$
\int_{-1 / 2}^{1 / 2} \cdots \int_{-1 / 2}^{1 / 2} g\left(\sum_{n=1}^{k q} a_{p_{j, n}}^{j} e_{p_{j, n}}\right) d a_{p_{j, 1}}^{j} \cdots d a_{p_{j, q k}}^{j}
$$

for functions $g$ on $\mathbb{R}^{d}$. 
Now rename the sequence

$$
a_{[1]}^{1}, a_{[2]}^{1}, \ldots, a_{[r]}^{1}, a_{[r+1]}^{2}, \ldots, a_{[2 r]}^{2}, \ldots, a_{[(k-1) r+1]}^{k}, \ldots, a_{[k r]}^{k}
$$

of variables in the second class as the sequence

$$
b_{1,1}, b_{1,2} \ldots, b_{1, k}, b_{2,1}, \ldots, b_{2, k}, \ldots, b_{r, 1}, \ldots, b_{r, k} .
$$

and similarly rename the sequence

$$
e_{[1]}, e_{[2]}, \ldots, e_{[r]}, e_{[r+1]}, \ldots, e_{[2 r]}, \ldots, e_{[(k-1) r+1]}, \ldots, e_{[k r]}
$$

of unit vectors as

$$
e_{q_{1,1}}, e_{q_{1,2}} \ldots, e_{q_{1, k}}, e_{q_{2,1}}, \ldots, e_{q_{2, k}}, \ldots, e_{q_{r, 1}}, \ldots, e_{q_{r, k}} .
$$

Since $k<d$ it is clear that for each $j=1,2, \ldots, r$ the $k$ unit vectors $e_{q_{j, 1}}, e_{q_{j, 2}} \ldots, e_{q_{j, k}}$ are distinct. For such $j$ define the measures $\lambda_{d-j+1}$ on $K_{0}$ by

$$
\int_{K_{0}} g\left(x_{d-j+1}\right) d \lambda_{d-j+1}\left(x_{d-j+1}\right)=\int_{-1 / 2}^{1 / 2} \cdots \int_{-1 / 2}^{1 / 2} g\left(\sum_{n=1}^{k} b_{j, n} e_{q_{j, n}}\right) d b_{j, 1} \cdots b_{j, k} .
$$

Let $\Pi_{2}$ represent the projection of $\left(\mathbb{R}^{d}\right)^{k}$ onto the $k r$-dimensional space corresponding to the variables of the second class and let $\Pi_{1}$ be the complementary projection. By the choice of the $n_{j}$ 's it follows that, if $f$ is a nonnegative function on $\left(\mathbb{R}^{d}\right)^{k}$, we have

$$
\int_{\left(K_{0}\right)^{r}} f\left(\Pi_{2} \circ \Psi^{\prime \prime}\left(x_{d-r+1}, \ldots, x_{d}\right)\right) d \lambda_{d-r+1}\left(x_{d-r+1}\right) \cdots d \lambda_{d}\left(x_{d}\right) \leq \int_{\Pi_{2}\left(\left(\mathbb{R}^{d}\right)^{k}\right)} f d m_{k r}
$$

(to see this, write $\left(x_{d}, \ldots, x_{d-r+1}\right)=\left(b_{1,1}, \ldots, b_{r, k}\right)$ and observe that the matrix of the map

$$
\left(b_{1,1}, \ldots, b_{r, k}\right) \mapsto \Pi_{2} \circ \Psi^{\prime \prime}\left(x_{d-r+1}, \ldots, x_{d}\right)
$$

is lower triangular with 1's on the diagonal). Since

$$
\int_{\left(K_{0}\right)^{q k}} f\left(\Psi^{\prime}\left(x_{1}, \ldots, x_{d-r}\right)\right) d \lambda_{1}\left(x_{1}\right) \cdots d \lambda_{d-r}\left(x_{d-r}\right)=\int_{\Pi_{1}([-1 / 2,1 / 2] d k)} f d m_{k(d-r)}
$$

it then follows that

$$
\int_{\left(K_{0}\right)^{d}} f\left(\Psi^{\prime}\left(x_{1}, \ldots, x_{d-r}\right)+\Psi^{\prime \prime}\left(x_{d-r+1}, \ldots, x_{d}\right)\right) d \lambda_{1}\left(x_{1}\right) \cdots d \lambda_{d}\left(x_{d}\right) \leq \int_{\left(\mathbb{R}^{d}\right)^{k}} f d m_{d k}
$$

giving (2.9) as desired.

The main result of this section is the following theorem.

Theorem 2.1. Suppose $K$ is a $k$-dimensional $C^{(1)}$ surface in $\mathbb{R}^{d}$ which is nondegenerate in the sense that $m_{d k}\left(\Psi\left(K^{d}\right)\right)>0$. Then (2.2) holds with $\alpha=k$ and so (1.4) holds as well.

The proof is an immediate consequence of the next two lemmas. 
Lemma 2.2. Suppose $K \subset \mathbb{R}^{d}$ carries probability measures $\lambda_{1}, \ldots \lambda_{d}$ such that

$$
\int_{K^{d}} f\left(\Psi\left(x_{1}, \ldots, x_{d}\right)\right) d \lambda_{1}\left(x_{1}\right) \cdots d \lambda_{d}\left(x_{d}\right) \lesssim \int_{\left(\mathbb{R}^{d}\right)^{k}} f d m_{d k}
$$

for nonnegative $f$ on $\mathbb{R}^{d}$. Then the estimate

$$
\int_{\mathbb{R}^{d}} \prod_{j=1}^{d} \int_{K} \chi_{E}\left(y+x_{j}\right) d \lambda_{j}\left(x_{j}\right) d m_{d}(y) \lesssim m_{d}(E)^{d /(d-k)}
$$

holds for Borel $E \subset \mathbb{R}^{d}$.

Lemma 2.3. If $K$ is as in Theorem 2.1 then $K$ carries probability measures $\lambda_{j}$ such that (2.10) holds.

To deduce (2.2) with $\alpha=k$ from the lemmas, fix $F$ and take $E=K+F$ in Lemma 2.2,

Here is the proof of Lemma 2.2.

Proof. The proof is based on ideas from [4] (see also [3]). Let $\Omega$ be defined by

$$
\Omega=\int_{\mathbb{R}^{d}} \prod_{j=1}^{d} \int_{K} \chi_{E}\left(y+x_{j}\right) d \lambda_{j}\left(x_{j}\right) d m_{d}(y)
$$

and put $\alpha=\Omega / m_{d}(E)$. It is enough to prove

$$
\alpha^{d-k} \lesssim m_{d}(E)^{k}
$$

Since

$$
\Omega=\int_{E} \int_{K} \prod_{j=2}^{d}\left(\int_{K} \chi_{E}\left(y+x_{j}-x_{1}\right) d \lambda_{j}\left(x_{j}\right)\right) d \lambda_{1}\left(x_{1}\right) d m_{d}(y),
$$

if $E_{1}$ is the set of $y \in E$ for which

$$
\int_{K} \prod_{j=2}^{d}\left(\int_{K} \chi_{E}\left(y+x_{j}-x_{1}\right) d \lambda_{j}\left(x_{j}\right)\right) d \lambda_{1}\left(x_{1}\right) \geq \frac{\alpha}{2}
$$

then

$$
\begin{aligned}
& \int_{\mathbb{R}^{d}} \int_{K} \chi_{E_{1}}\left(y+x_{1}\right) d \lambda_{1}\left(x_{1}\right) \prod_{j=2}^{d} \int_{K} \chi_{E}\left(y+x_{j}\right) d \lambda_{j}\left(x_{j}\right) d m_{d}(y)= \\
& \int_{E_{1}} \int_{K} \prod_{j=2}^{d}\left(\int_{K} \chi_{E}\left(y+x_{j}-x_{1}\right) d \lambda_{j}\left(x_{j}\right)\right) d \lambda_{1}\left(x_{1}\right) d m_{d}(y) \geq \frac{\Omega}{2} .
\end{aligned}
$$


Continuing inductively, for $j=2, \ldots d$ we produce nonempty sets $E_{j} \subset E$ such that

$$
\int_{K}\left(\prod_{p<j} \int_{K} \chi_{E_{p}}\left(y+x_{j}-x_{p}\right) d \lambda_{p}\left(x_{p}\right) \prod_{q>j} \int_{K} \chi_{E}\left(y+x_{j}-x_{q}\right) d \lambda_{q}\left(x_{q}\right)\right) d \lambda_{j}\left(x_{j}\right) \geq \frac{\alpha}{2^{j}}
$$

for $y \in E_{j}$.

We will make repeated use of the following observation (a consequence of (2.13) ): if $j=2, \ldots, d$ then

$$
\int_{K}\left(\int_{K} \prod_{p=1}^{j-1} \chi_{E_{p}}\left(y+x_{j}-x_{p}\right) d \lambda_{p}\left(x_{p}\right)\right) d \lambda_{j}\left(x_{j}\right) \gtrsim \alpha \chi_{E_{j}}(y) .
$$

Here are some notational conventions which we will use in the remainder of the proof. The symbol $\mathbb{E}$ will denote an expectation and any subscripted $y$ will denote a random vector in $\mathbb{R}^{d}$. The underlying probability space will be the product of a large number of copies of $K$ with probability measure a product of measures $\lambda_{j}$. Thus, with $y_{p}=-x_{p}$, we rewrite (2.14) as

$$
\mathbb{E}\left(\int_{K} \prod_{p=1}^{j-1} \chi_{E_{p}}\left(y+y_{p}+x_{j}\right) d \lambda_{j}\left(x_{j}\right)\right) \gtrsim \alpha \chi_{E_{j}}(y) .
$$

In particular, starting with $y \in E_{d}$ and then writing $y_{p}^{1}=y+y_{p}$, we have

$$
\mathbb{E}\left(\int_{K} \prod_{p=1}^{d-1} \chi_{E_{p}}\left(y_{p}^{1}+x_{d}\right) d \lambda_{d}\left(x_{d}\right)\right) \gtrsim \alpha
$$

Now, since

$\mathbb{E}_{\left\{y_{p}^{2}\right\}}\left(\int_{K} \prod_{p=1}^{d-2} \chi_{E_{p}}\left(y_{d-1}^{1}+y_{p}^{2}+x_{d}+x_{d-1}\right) d \lambda_{d-1}\left(x_{d-1}\right)\right) \gtrsim \alpha \chi_{E_{d-1}}\left(y_{d-1}^{1}+x_{d}\right)$

by (2.15), we see, upon replacing $y_{d-1}^{1}+y_{p}^{2}$ by $y_{p}^{2}$, that (2.16) yields

$\mathbb{E}\left(\int_{K} \int_{K} \prod_{p_{1}=1}^{d-1} \chi_{E_{p_{1}}}\left(y_{p_{1}}^{1}+x_{d}\right) \prod_{p_{2}=1}^{d-2} \chi_{E_{p_{2}}}\left(y_{p_{2}}^{2}+x_{d}+x_{d-1}\right) d \lambda_{d}\left(x_{d}\right) d \lambda_{d-1}\left(x_{d-1}\right)\right) \gtrsim \alpha^{2}$.

Next use (2.15) again to write

$$
\mathbb{E}_{\left\{y_{p}\right\}}\left(\int_{K} \prod_{p=1}^{d-3} \chi_{E_{p}}\left(y_{p}+y_{d-2}^{2}+x_{d}+x_{d-1}+x_{d-2}\right) d \lambda_{d-2}\left(x_{d-2}\right)\right) \gtrsim \alpha \chi_{E_{d-2}}\left(y_{d-2}^{2}+x_{d}+x_{d-1}\right)
$$

and apply this to (2.17) to obtain

$$
\mathbb{E}\left(\int_{K} \int_{K} \int_{K} \prod_{p=1}^{3}\left[\prod_{q=1}^{d-p} \chi_{E_{q}}\left(y_{p, q}+x_{d}+\cdots+x_{d-p+1}\right)\right] d \lambda_{d}\left(x_{d}\right) d \lambda_{d-1}\left(x_{d-1}\right) d \lambda_{d-2}\left(x_{d-2}\right)\right) \gtrsim \alpha^{3} .
$$


After $r-3$ more steps (where we recall that $r$ is defined by $d=q k+r$ ), we have

$$
\mathbb{E}\left(\int_{K} \cdots \int_{K} \prod_{p=1}^{r}\left[\prod_{q=1}^{d-p} \chi_{E_{q}}\left(y_{p, q}+x_{d}+\cdots+x_{d-p+1}\right)\right] d \lambda_{d}\left(x_{d}\right) \cdots d \lambda_{d-r+1}\left(x_{d-r+1}\right)\right) \gtrsim \alpha^{r} .
$$

We make another notational convention: $\int \cdots d \lambda(x)$ will stand for an integral over a product of copies of $K$ with respect to a product of measures $\lambda_{j}$ where the measures occurring in the product correspond to the variables $x_{j}$ appearing in the integrand. In particular we rewrite (2.18) as

$$
\mathbb{E}\left(\int \prod_{p=1}^{r}\left[\prod_{q=1}^{d-p} \chi_{E_{q}}\left(y_{p, q}+x_{d}+\cdots+x_{d-p+1}\right)\right] d \lambda(x)\right) \gtrsim \alpha^{r} .
$$

Now fix $j \in\{1, \ldots, k\}$. With $n_{j}$ as specified after (2.6), (2.15) gives

$$
\mathbb{E}\left(\int_{K} \chi_{E_{j q-1}}\left(y_{j}+x_{d}+\cdots+x_{d-n_{j}}+x_{j q}\right) d \lambda_{j q}\left(x_{j q}\right)\right) \gtrsim \alpha \chi_{E_{j q}}\left(y_{n_{j}+1, j q}+x_{d}+\cdots+x_{d-n_{j}}\right)
$$

for some random vector $y_{j}$. Since $n_{j}<r$ implies $j q \leq k q=d-r<d-n_{j}$, the $k$ estimates (2.20) can be applied in (2.19) to give

$$
\mathbb{E}\left(\int \prod_{j=1}^{k} \chi_{E_{j q-1}}\left(y_{j}+x_{d}+\cdots+x_{d-n_{j}}+x_{j q}\right) d \lambda(x)\right) \gtrsim \alpha^{r+k} .
$$

Analogous to (2.20) there are, for $1 \leq j \leq k$, the estimates

$$
\begin{gathered}
\mathbb{E}\left(\int_{K} \chi_{E_{j q-2}}\left(y_{j}^{\prime}+x_{d}+\cdots+x_{d-n_{j}}+x_{j q}+x_{j q-1}\right) d \lambda_{j q-1}\left(x_{j q-1}\right)\right) \\
\gtrsim \alpha \chi_{E_{j q-1}}\left(y_{j}+x_{d}+\cdots+x_{d-n_{j}}+x_{j q}\right) .
\end{gathered}
$$

Using these estimates in (2.21) gives

$$
\mathbb{E}\left(\int \prod_{j=1}^{k} \chi_{E_{j q-2}}\left(y_{j}^{\prime}+x_{d}+\cdots+x_{d-n_{j}}+x_{j q}+x_{j q-1}\right) d \lambda(x)\right) \gtrsim \alpha^{r+2 k} .
$$

Doing this $q-4$ more times we obtain

$$
\mathbb{E}\left(\int \prod_{j=1}^{k} \chi_{E_{(j-1) q+2}}\left(y_{j}+x_{d}+\cdots+x_{d-n_{j}}+x_{j q}+x_{j q-1}+\cdots+x_{(j-1) q+3}\right) d \lambda(x)\right) \gtrsim \alpha^{r+(q-2) k} .
$$

Recall from (2.14) that

$$
\int_{K} \int_{K} \chi_{E_{p-1}}\left(y+x_{p}-x_{p-1}\right) d \lambda_{p}\left(x_{p}\right) d \lambda_{p-1}\left(x_{p-1}\right) \gtrsim \alpha \chi_{E_{p}}(y)
$$


and apply this with $p=(j-1) q+2$ to obtain the estimates

$$
\begin{gathered}
\int_{K} \int_{K} \chi_{E_{(j-1) q+1}}\left(y_{j}+x_{d}+\cdots+x_{d-n_{j}}+x_{j q}+\cdots+x_{(j-1) q+2}-x_{(j-1) q+1}\right) . \\
d \lambda_{(j-1) q+2}\left(x_{(j-1) q+2}\right) d \lambda_{(j-1) q+1}\left(x_{(j-1) q+1}\right) \\
\gtrsim \alpha \chi_{E_{(j-1) q+2}}\left(y_{j}+x_{d}+\cdots+x_{d-n_{j}}+x_{j q}+\cdots+x_{(j-1) q+3}\right) .
\end{gathered}
$$

Using these in (2.22) and recalling the definition of $\psi_{j}$ gives

$$
\mathbb{E}\left(\int \prod_{j=1}^{k} \chi_{E_{(j-1) q+1}}\left(y_{j}+\psi_{j}\left(x_{1}, \ldots, x_{d}\right)\right) d \lambda(x)\right) \gtrsim \alpha^{r+(q-1) k}
$$

and so, since $E_{i} \subset E$ and $r+(q-1) k=d-k$,

$$
\mathbb{E}\left(\int \prod_{j=1}^{k} \chi_{E}\left(y_{j}+\psi_{j}\left(x_{1}, \ldots, x_{d}\right)\right) d \lambda(x)\right) \gtrsim \alpha^{d-k} .
$$

Now applying the hypothesis (2.10) with $f$ the indicator function of

$$
\prod_{j=1}^{k}\left(E-y_{j}\right) \subset\left(\mathbb{R}^{d}\right)^{k}
$$

yields (2.12), completing the proof of Lemma 2.2,

The proof of Lemma 2.3 is the same as the analogous part of the proof of Theorem 3 in [11, but for the sake of completeness (and since it is short), we will sketch the argument: parametrize $K$ by a $C^{(1)}$ map $\phi:(0,1)^{k} \rightarrow \mathbb{R}^{d}$. Let $\Phi:\left((0,1)^{k}\right)^{d} \rightarrow\left(\mathbb{R}^{d}\right)^{k}$ be defined by

$$
\Phi\left(x_{1}, \ldots, x_{d}\right)=\Psi\left(\phi\left(x_{1}\right), \ldots, \phi\left(x_{d}\right)\right) .
$$

The hypothesis is that $\Phi\left(\left((0,1)^{k}\right)^{d}\right)$ has positive Lebesgue measure in $\left(\mathbb{R}^{d}\right)^{k}$. It follows from Sard's theorem, continuity, and the inverse function theorem, that there are $\delta>0$ and nonempty open sets $O_{j} \subset(0,1)^{k}$ such that

$$
\left|\operatorname{det} \Phi^{\prime}\left(x_{1}, \ldots, x_{d}\right)\right| \geq \delta
$$

on $\prod_{j=1}^{d} O_{j}$ and such that $\Phi$ is one-to-one on $\prod_{j=1}^{d} O_{j}$. If the measures $\lambda_{j}$ are defined by

$$
\int_{K} g d \lambda_{j}=\frac{1}{m_{k}\left(O_{j}\right)} \int_{O_{j}} g(\phi(x)) d m_{k}(x)
$$

then it is easy to see that the $\lambda_{j}$ 's satisfy the conclusion of Lemma 2.3 . 


\section{HAUSDORFF DIMENSION}

One approach to studying $\operatorname{dim}_{h}(E+K)$ begins with the fact that if $K$ and $E$ are, respectively, the supports of measures $\lambda$ and $\mu$, then $K+E$ is the support of $\lambda * \mu$. An attempt to exploit this idea might start with Frostman measures on $K$ and $E$ and hope to say something useful about the energies of $\lambda * \mu$. In our context, with $K$ fixed and desiring to estimate $\operatorname{dim}_{h}(E+K)$ for general $E$, it seems necessary to require more than that $\lambda$ be a Frostman measure for $K$. If we require much more, namely that $K$ be a Salem set, then it is easy to show that

$$
\operatorname{dim}_{h}(E+K)=\min \left\{\operatorname{dim}_{h}(E)+\operatorname{dim}_{h}(K), d\right\} .
$$

Recall that a set $K \subset \mathbb{R}^{d}$ satisfying $\operatorname{dim}_{h}(K)=\alpha$ is a Salem set if, for each $s<\alpha, K$ carries a probability measure $\lambda$ satisfying

$$
|\widehat{\lambda}(\xi)| \lesssim|\xi|^{-s / 2}
$$

(Kahane's book 5 is a good source of information about Salem sets.) Suppose that $K$ is a Salem set and also that $\operatorname{dim}_{h}(E)=\beta$. Now $\operatorname{dim}_{h}(E)=\beta$ is

equivalent to the statement that if $r<\beta$ then there is a probability measure $\mu$ supported on $E$ such that

$$
\int_{\mathbb{R}^{d}}|\xi|^{r-d}|\widehat{\mu}(\xi)|^{2} d \xi<\infty
$$

If (3.2) holds, then (3.3) gives

$$
\int_{\mathbb{R}^{d}}|\xi|^{r+s-d}|\widehat{\lambda * \mu}(\xi)|^{2} d \xi<\infty .
$$

Thus whenever $r<\beta$ and $s<\alpha, E+K$ carries a probability measure $\nu$ such that

$$
\int_{\mathbb{R}^{d}}|\xi|^{r+s-d}|\widehat{\nu}(\xi)|^{2} d \xi<\infty
$$

and (3.1) follows. Of course this argument shows that if $K$ carries a probability measure $\lambda$ satisfying (3.2) then

$$
\operatorname{dim}_{h}(E+K) \geq \min \left\{\operatorname{dim}_{h}(E)+s, d\right\} .
$$

Unfortunately, the requirement that $K$ be a Salem set is stringent: for example, a $k$-surface in $\mathbb{R}^{d}$ can be a Salem set only if $k=d-1$.

Here is a connection between the theory of $L^{p} \rightarrow L^{q}$ convolution estimates for nonnegative measures $\lambda$ and estimates for $\operatorname{dim}_{h}(E+K)$ :

Proposition 3.1. Suppose $\lambda$ is a probability measure on $K \subset \mathbb{R}^{d}$ which satisfies the convolution estimate

$$
\|\lambda * f\|_{L^{q}\left(\mathbb{R}^{d}\right)} \lesssim\|f\|_{L^{p}\left(\mathbb{R}^{d}\right)}
$$

for indices $1<p<q<\infty$. If $\operatorname{dim}_{h}(E)=\beta$ then

$$
\operatorname{dim}_{h}(K+E) \geq q^{\prime}\left[\frac{d}{p}-\frac{d}{q}+\frac{\beta}{p^{\prime}}\right]
$$


Before giving the proof we make some comments:

(i) If (3.5) holds, then convolution with the characteristic function of a small ball shows that $(1 / p, 1 / q)$ must lie in the triangle $\Delta(\alpha, d)$ with vertices $(0,0),(1,1)$, and $(d /(2 d-\alpha),(d-\alpha) /(2 d-\alpha))$, where $\alpha$ is the Hausdorff dimension of the support of $\lambda$. If $\lambda$ is supported on a $k$-surface in $\mathbb{R}^{d}$ and $k(k+3)<2 d$, then (3.5) implies additional necessary conditions which keep $(1 / p, 1 / q)$ bounded away from $(d /(2 d-\alpha),(d-\alpha) /(2 d-\alpha))$. But if, for example, $k>d / 2$, then there are $k$-surfaces such that (3.5) holds for all $(1 / p, 1 / q)$ in the interior of the triangle $\Delta(k, d)$. See [8].

(ii) If (3.5) holds for all $(1 / p, 1 / q)$ in the interior of the triangle $\Delta(k, d)$, then it follows from Proposition 3.1 that the analog

$$
\operatorname{dim}_{h}(E+K) \geq \operatorname{dim}_{h}(K)+\operatorname{dim}_{h}(E)-\frac{\operatorname{dim}_{h}(K) \operatorname{dim}_{h}(E)}{d}
$$

of (1.4) holds.

(iii) If (3.5) holds, then

$m_{d}(F)=\int_{\mathbb{R}^{d}} \lambda * \chi_{F} d m_{d} \leq m_{d}(F+K)^{1 / q^{\prime}}\left\|\lambda * \chi_{F}\right\|_{L^{q}\left(\mathbb{R}^{d}\right)} \lesssim m_{d}(F+K)^{1 / q^{\prime}} m_{d}(F)^{1 / p}$

yields

$$
m_{d}(F)^{q^{\prime} / p^{\prime}} \lesssim m_{d}(F+K),
$$

unless $q=1$. In particular, if $(1 / p, 1 / q)$ lies on the open segment joining $(1,1)$ and $(d /(2 d-\alpha),(d-\alpha) /(2 d-\alpha))$, (3.7) is (2.2). (We deduced inequalities of the form (2.2) from Lemma 2.2. If the measures $\lambda_{j}$ in Lemma 2.2 are all equal, then the conclusion of Lemma 2.2 is an estimate like (3.5).)

(iv) If $\lambda$ is the (middle thirds) Cantor-Lebesgue measure on $\mathbb{R}$, then (3.5) holds for $(1 / p, 1 / q)=(2 / 3,1 / 3)$. Thus Proposition 3.1 yields $\operatorname{dim}_{h}(E+K) \geq$ $\left(\operatorname{dim}_{h}(E)+1\right) / 2$ for the Cantor set $K$. This improves the trivial estimate for $\operatorname{dim}_{h}(E+K)$ only if $\operatorname{dim}_{h}(E)>2 \log 2 / \log 3-1$.

Here is the proof of Proposition 3.1] (The material through Lemma 3.2 is, for the reader's convenience, repeated from [9].) For $\rho>0$, let $K_{\rho}$ be the kernel defined on $\mathbb{R}^{d}$ by $K_{\rho}(x)=|x|^{-\rho} \chi_{B(0, R)}(x)$ where $R=R(d)$ is positive. Suppose that the finite nonnegative Borel measure $\nu$ is a $\gamma$ dimensional measure on $\mathbb{R}^{d}$ in the sense that $\nu(B(x, \delta)) \leq C(\nu) \delta^{\gamma}$ for all $x \in \mathbb{R}^{d}$ and $\delta>0$. If $\rho<\gamma$ it follows that

$$
\nu * K_{\rho} \in L^{\infty}\left(\mathbb{R}^{d}\right) .
$$

Also

$$
\nu * K_{\rho} \in L^{1}\left(\mathbb{R}^{d}\right)
$$

so long as $\rho<d$. Thus, for $\epsilon>0$,

$$
\nu * K_{\rho} \in L^{p}\left(\mathbb{R}^{d}\right), \rho=\gamma+\frac{1}{p}(d-\gamma)-\epsilon
$$

by interpolation. The following lemma is a weak converse of this observation. 
Lemma 3.2. If (3.8) holds with $\epsilon=0$ and $p>1$, then $\nu$ is absolutely continuous with respect to Hausdorff measure of dimension $\gamma-\epsilon$ for any $\epsilon>0$. Thus the support of $\nu$ has Hausdorff dimension at least $\gamma$.

Proof. Recall from [1] (see p. 140) that, for $s \in \mathbb{R}$ and $1 \leq p, q \leq \infty$, the norm $\|f\|_{p, q}^{s}$ of a distribution $f$ on $\mathbb{R}^{d}$ in the Besov space $B_{p, q}^{s}$ can be defined by

$$
\|f\|_{p q}^{s}=\|\psi * f\|_{L^{p}\left(\mathbb{R}^{d}\right)}+\left(\sum_{k=1}^{\infty}\left(2^{s k}\left\|\phi_{k} * f\right\|_{L^{p}\left(\mathbb{R}^{d}\right)}\right)^{q}\right)^{1 / q}
$$

for certain fixed $\psi \in \mathcal{S}\left(\mathbb{R}^{d}\right), \phi \in C_{c}^{\infty}\left(\mathbb{R}^{d}\right)$, and where $\phi_{k}(x)=2^{k d} \phi\left(2^{k} x\right)$. If $\nu * K_{\rho} \in L^{p}\left(\mathbb{R}^{d}\right)$, then $\left\|\nu * \chi_{B(0, \delta)}\right\|_{L^{p}\left(\mathbb{R}^{d}\right)} \lesssim \delta^{\rho}$. It follows that $\|\nu\|_{p q}^{s}<\infty$ if $s<\rho-d=(\gamma-d) / p^{\prime}$. Now, for $t>0$ and $1<p^{\prime}, q^{\prime}<\infty$, the Besov capacity $A_{t, p^{\prime}, q^{\prime}}(K)$ of a compact $K \subset \mathbb{R}^{d}$ is defined by

$$
A_{t, p^{\prime}, q^{\prime}}(K)=\inf \left\{\|f\|_{p^{\prime}, q^{\prime}}^{t}: f \in C_{c}^{\infty}\left(\mathbb{R}^{d}\right), f \geq \chi_{K}\right\} .
$$

It is shown in [15] (see p. 277) that $A_{t, p^{\prime}, q^{\prime}}(K) \lesssim H_{d-t p^{\prime}}(K)$. Thus it follows from the duality of $B_{p, q}^{s}$ and $B_{p^{\prime}, q^{\prime}}^{-s}$ that

$$
\nu(K) \lesssim\|\nu\|_{p q}^{s} A_{-s, p^{\prime}, q^{\prime}}(K) \lesssim H_{d+s p^{\prime}}(K)=H_{\gamma-\epsilon}(K)
$$

if $s=(\gamma-d-\epsilon) / p^{\prime}$.

Now to prove Proposition [3.1, assume that $E \subset \mathbb{R}^{d}$ satisfies $\operatorname{dim}_{h}(E)=\beta$. Then, if $\epsilon>0, E$ supports a probability measure $\mu$ such that $\mu * K_{\rho} \epsilon$ $L^{p}\left(\mathbb{R}^{d}\right)$ where $\rho=\beta+(d-\beta) / p-\epsilon$. The hypothesis (3.5) implies that $\lambda * \mu * K_{\rho} \in L^{q}\left(\mathbb{R}^{d}\right)$. Then, since $\mu * \lambda$ is supported on $E+K$, Lemma 3.2 implies that

$$
\operatorname{dim}_{h}(E+K) \geq q^{\prime}\left[\frac{d}{p}-\frac{d}{q}+\frac{\alpha}{p^{\prime}}\right]-q^{\prime} \epsilon .
$$

We conclude this paper with a short discussion of the situation when $K$ is a curve in $\mathbb{R}^{d}$, and we begin with the case $d=2$. If $A \subset \mathbb{R}$ satisfies $\operatorname{dim}_{h}(A \times A)>2 \operatorname{dim}_{h}(A)$ then taking $K=K_{0}$, where $K_{0}$ is the polygonal curve mentioned after (1.3), and $E=A \times A$ shows that the analog for Hausdorff dimension of (1.3) can fail for curves which satisfy only the weak nondegeneracy requirement of $\oint_{2}$. Things are much improved if we require that $K$ be a $C^{(2)}$ curve in $\mathbb{R}^{2}$ (and the situation is analogous for hypersurfaces in $\mathbb{R}^{d}$ ): then, if $K$ is not a line segment - i.e., if $K$ is not degenerate in the context of the problem at hand - the arclength measure $\lambda$ on an interval of $K$ where the curvature does not vanish will satisfy $|\widehat{\lambda}(\xi)| \lesssim|\xi|^{-1 / 2}, K$ will be a Salem set, and so we will have, by (3.1),

$$
\operatorname{dim}_{h}(E+K) \geq \min \left\{\operatorname{dim}_{h}(E)+1,2\right\}, E \subset \mathbb{R}^{2} .
$$

An analog for $\mathbb{R}^{3}$ of (3.9) is also not difficult to establish. But the argument depends on deep results from [13] about the Sobolev mapping 
properties of averaging operators associated with certain curves in $\mathbb{R}^{3}$. Let $K=\{\gamma(t): 0<t<1\} \subset \mathbb{R}^{3}$ be a curve $\{\gamma(t): 0 \leq t \leq 1\}$ of finite type (see 13 for the definition) and let $\lambda$ be the measure induced on $K$ by setting $d \lambda=d t$. Suppose that $0<\beta \leq 2$ and that $\mu$ is a Borel measure on $E$ which is $\beta$-dimensional in the sense that $\mu(B(x, \delta)) \lesssim \delta^{\beta}$ for $\delta>0, x \in \mathbb{R}^{3}$. Fix $\epsilon>0$. We will show that

$$
\delta^{-3}\left\langle\chi_{B(0, \delta)} * \mu * \lambda, \chi_{F}\right\rangle \lesssim \delta^{-[3-(1+\beta)+\epsilon] / p} m_{3}(F)^{1 / p}
$$

for some $p$, all Borel $F \subset \mathbb{R}^{3}$, and all $\delta>0$. By a well known argument from [2] it will then follow that

$$
\operatorname{dim}_{h}(E+K) \geq \min \{\beta+1,3\}, E \subset \mathbb{R}^{3} .
$$

With $\|\cdot\|_{p, s}$ denoting the norm of the $L^{p}$ Sobolev space $L_{s}^{p}\left(\mathbb{R}^{3}\right)$, Theorem 1.1 in [13] furnishes $p$ such that there is the convolution estimate

$$
\|\lambda * f\|_{p, 1 / p} \lesssim\|f\|_{L^{p}\left(\mathbb{R}^{3}\right)} .
$$

On the other hand, (3.8) shows that

$$
\mu * K_{\rho} \in L^{p^{\prime}}\left(\mathbb{R}^{3}\right) \text { if } \rho-3=\frac{\beta-3}{p}-\epsilon
$$

and so

$$
\mu * K_{\rho} \in L_{-1 / p}^{p^{\prime}}\left(\mathbb{R}^{3}\right) \text { if } \rho-3=\frac{\beta-2}{p}-\epsilon .
$$

Taking $f=\chi_{F}$ in (3.12) and $\rho=3+\frac{\beta-2}{p}-\epsilon$, this gives

$$
\left\langle K_{\rho} * \mu * \lambda, \chi_{F}\right\rangle \lesssim m_{3}(F)^{1 / p} .
$$

Since $\delta^{-\rho} \chi_{B(0, \delta)} \lesssim K_{\rho},(3.10)$ follows.

One can hope that the analog of (3.11) persists for nondegenerate curves in $\mathbb{R}^{d}$ when $d>3$. But there is currently no result like Theorem 1.1 in [13] available in higher dimensions, and we can only make a few observations. If $d \lambda$ is $d t$ on a segment $K$ of the model curve $\left(t, t^{2}, \ldots, t^{d}\right)$ in $\mathbb{R}^{d}$, then the best estimate for $\widehat{\lambda}$ is $|\widehat{\lambda}(\xi)| \lesssim|\xi|^{-1 / d}$. Thus (3.4) yields only

$$
\operatorname{dim}_{h}(E+K) \geq \min \left\{\operatorname{dim}_{h}(E)+2 / d, d\right\} .
$$

And, using Christ's theorem from [4] about the $L^{p} \rightarrow L^{q}$ convolution properties of $\lambda$, Proposition 3.1 gives

$$
\operatorname{dim}_{h}(E+K) \geq \min \left\{(1-1 / d) \operatorname{dim}_{h}(E)+1, d\right\} .
$$

On the other hand, given (3.11) it is easy to see that if $E \subset \mathbb{R}^{d}$ satisfies $\operatorname{dim}_{h}(E) \leq 2$, then

$$
\operatorname{dim}_{h}(E+K) \geq \operatorname{dim}_{h}(E)+1 .
$$

This is because Marstrand's projection theorem [7] implies that for almost all orthogonal projections $\pi$ of $\mathbb{R}^{d}$ onto a three-dimensional subspace we 
have $\operatorname{dim}_{h}(\pi(E))=\operatorname{dim}_{h}(E)$. Choose such a $\pi$ and note that, by (3.11) and the fact that $\pi(K)$ is of finite type,

$\operatorname{dim}_{h}(E+K) \geq \operatorname{dim}_{h}(\pi(E+K))=\operatorname{dim}_{h}(\pi(E)+\pi(K)) \geq \operatorname{dim}_{h}(\pi(E))+1$.

\section{REFERENCES}

[1] J. Bergh, J. Löfström, Interpolation Spaces, Grundlehren der mathematischen Wissenschaften, bd. 223, Springer-Verlag, Berlin, 1976.

[2] J. Bourgarin, Besicovitch type maximal operators and applications to Fourier analysis, Geom. Funct. Anal. 1 (1991), 147-187.

[3] M. Christ, Counting to $L^{p}$, Lecture notes for the Instructional Conference on Combinatorial Aspects of Mathematical Analysis, ICMS, Edinburgh, April 1-2, 2002.

[4] Convolution, curvature, and combinatorics: case study, Internat. Math. Research Notices 19 (1998), 1033-1048.

[5] J.-P. Kahane, Some random series of functions, 2nd ed., Cambridge Studies in Advanced Mathematics, Cambridge University Press, Cambridge, 1985.

[6] R. Kaufman, On Hausdorff dimension of projections, Mathematika 15 (1968), 153155.

[7] J. Marstrand, Some fundamental geometrical properties of plane sets of fractional dimension, Proc. London. Math. Soc. 4 (1954), 257-302.

[8] D. Oberlin, Convolution estimates and model surfaces of low codimension, J. Fourier Anal. Appl. 14 (2008), 484-491. preprint.

[9] Restricted Radon transforms and projections of planar sets, preprint.

[10] The size of sums of sets, Studia Math. 83 (1986), 139-146.

[11] The size of sums of sets, II, Israel J. of Math. 55 (1986), 305-316.

[12] Y. Peres and P. Shmerkin, Resonance between Cantor sets, preprint.

[13] M. Praminik and A. Seeger Averages over curves in $\mathbb{R}^{3}$ and associated maximal functions, Amer. J. Math. 21 (2007), 61-103.

[14] P. Shmerkin, Moreira's theorem on the arithmetic sum of dynamically defined Cantor sets, preprint.

[15] B.-M. Stocke, Differentiability properties of Besov potentials and Besov spaces, Arkiv för Mat. 22 (1984), 269-286.

[16] T. Wolff, Recent work connected with the Kakeya problem, Prospects in Mathematics (Princeton, NJ, 1966), Amer. Math. Soc., Providence, RI, (1966), 129-162.

D. M. Oberlin, Department of Mathematics, Florida State University, TalLAHASSEE, FL 32306

E-mail address: oberlin@math.fsu.edu 